\title{
Role of edema in peritumoral cyst formation
}

\author{
Martin A. Baggenstos, M.D., ${ }^{1,2}$ John A. Butman, M.D., Ph.D., ${ }^{3}$ Edward H. Oldfield, \\ M.D., ${ }^{1}$ AND RUSSELl R. LONSER, M.D. ${ }^{1}$
${ }^{1}$ Surgical Neurology Branch, National Institute of Neurological Disorders and Stroke; ${ }^{3}$ Section of Neuroradiology, Diagnostic Radiology Department, The Clinical Center of the National Institutes of Health, Bethesda, Maryland; and ${ }^{2}$ Department of Neurosurgery, George Washington University, Washington, D.C.

\begin{abstract}
$\checkmark$ Peritumoral cysts (those arising immediately adjacent to the tumor mass) are frequently associated with benign and malignant tumors of the brain and spinal cord (syringomyelia). The cystic component of central nervous system (CNS) tumors and associated peritumoral cysts are often the cause of clinical symptoms. Because of the common occurrence of peritumoral cysts with CNS neoplasms and the morbidity associated with them, advanced imaging, histological, and molecular techniques have been used to determine the mechanism underlying cyst formation and propagation. Based on evidence from such studies, edema appears to be a common precursor to peritumoral cyst formation in the CNS. Mediators of vascular permeability acting locally in the tumor and/or hydrodynamic forces within abnormal tumor vasculature appear to drive fluid extravasation. When these forces overcome the ability of surrounding tissue to resorb fluid, edema and subsequent cyst formation occur. These findings support the concept that the tumor itself is the source of the edema that precedes cyst formation and that resection of tumors or medical therapies directed at decreasing their vascular permeability will result in the resolution of edema and cysts.
\end{abstract}

KeY WordS • cyst • edema - mechanism • neurooncology • pathophysiology • tumor

$\mathrm{T}$ WO TYPES OF cysts-peritumoral and intratumoralare associated with CNS tumors. Peritumoral cysts develop within the brain or spinal cord and form at the margin of the tumor. Alternatively, intratumoral cysts develop within the tumor itself and are usually the result of intratumoral necrosis. ${ }^{1}$ Overall, cysts are associated with approximately $10 \%$ of benign, malignant, and metastatic tumors of the CNS. ${ }^{1,26}$ They are most frequently associated with hemangioblastomas $(83 \%)$, cerebellar astrocytomas (77\%), and cerebral astrocytomas (29\%). ${ }^{24,26}$ The presence of peritumoral cysts can lead to significant neurological impairment due to mass effect and increased intracranial pressure. Based on advances in imaging, histological, and molecular techniques, insight into the mechanism behind peritumoral cyst formation has been provided, and evidence indicates that peritumoral edema precedes and underlies the propagation of these cysts.

Abbreviations used in this paper: $\mathrm{CNS}=$ central nervous system; $\mathrm{CSF}=$ cerebrospinal fluid; DTPA = diethylenetriamine pentaacetic acid; $\mathrm{GBM}=$ glioblastoma multiforme; $\mathrm{MR}=$ magnetic resonance; VEGF = vascular endothelial growth factor.

\section{Edema and Peritumoral Cyst Formation}

\section{General Mechanism}

Emerging data show that peritumoral cysts form through the extravasation of a plasma ultrafiltrate through tumor vessels with increased vascular permeability. ${ }^{11,24,27,32}$ The increase in tumor vessel permeability leads to extravasation of a plasma ultrafiltrate into the interstitial space of the tumor. ${ }^{11,24,27,32}$ The high interstitial pressure within the lesion, in relation to the interstitial pressure of surrounding brain, acts as a convective force that drives the extracellular plasma ultrafiltrate from the interstitial space of the tumor to that of the surrounding brain or spinal cord. $5,17,32$ Initially, the extravasation of fluid produces a small region of edema surrounding the tumor. Equilibrium is achieved: excess fluid produced by the tumor is absorbed by surrounding edematous tissues. As time passes, increasing vessel surface area and permeability within the neoplasm along with increasing intratumoral pressure delivers increasing volumes of interstitial fluid to surrounding tissue. Furthermore, reactive gliosis and increased interstitial pressure within surrounding tissue reduces its capacity to absorb the interstitial fluid. Thus, 

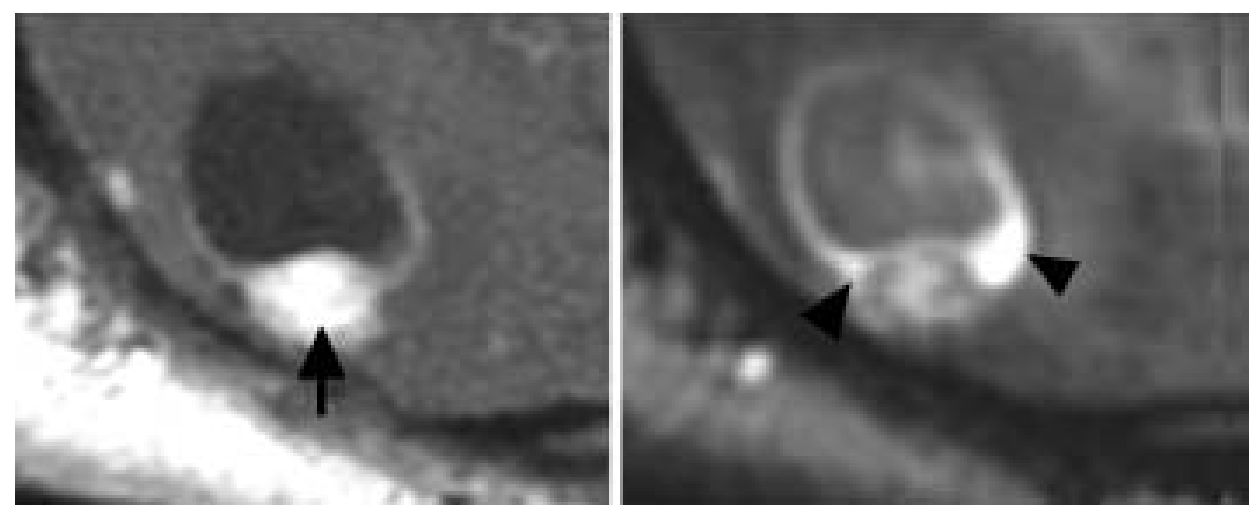

FIG. 1. Axial images obtained through the posterior fossa in a patient with a cerebellar hemangioblastoma, demonstrating leakage of contrast agent into the tumor-associated cyst. Left: Contrast-enhanced T1-weighted MR image revealing enhancement of a hemangioblastoma (arrow). Right: Corresponding contrast-enhanced fluid attenuated inversion recovery image showing extravasation of the agent (arrowheads) into an associated cyst and settling in its dependent portions.

the volume of tissue affected by interstitial edema increases. Over time, when absorption exceeds the extravasation of plasma ultrafiltrate, surrounding tissue will swell due to its subjection to solid stresses (stretching) that favor cyst formation..$^{24,27,32}$ This process results in the formation of a peritumoral cyst. Formation of the cyst alters interstitial flow patterns so that the majority of excess interstitial fluid channels into the peritumoral cyst, given that it is the pathway of least flow resistance. ${ }^{28,32}$ We describe the clinical, neuroimaging, histological, and molecular evidence that underlies this mechanism of peritumoral cyst formation.

\section{Peritumoral Edema Underlies Peritumoral Cyst Formation}

Neuroimaging Evidence. Consistent with the origination of peritumoral edema from highly permeable tumor vessels and an association between edema and peritumoral cyst formation, MR imaging and computed tomography study data have demonstrated that increased tumor vascular permeability is associated with increases in peritumoral edema, which in turn is associated with peritumoral cyst formation. Pronin and colleagues ${ }^{39}$ and Holodny and associates $^{16}$ found that increased tumor vessel permeability, as demonstrated by increased enhancement on MR images, directly correlated with increasing peritumoral edema in gliomas. Lohle and coworkers ${ }^{23}$ extended these findings and used MR imaging and computed tomography volumetric analysis (tumor, tissue edema, and peritumoral cyst volumes) to determine the relationship between tumorassociated edema and peritumoral cysts. Results of an analysis of 60 patients harboring either primary (30 patients) or metastatic (30 patients) brain neoplasms (half of the tumors had associated peritumoral cysts) revealed that lesions with a larger tumor/edema volume ratio were significantly more likely to have an associated peritumoral cyst, indicating a link between tumor-associated edema and peritumoral cyst formation.

Consistent with edema and cyst formation due to plasma extravasation from permeable tumor vessels, dynamic contrast-enhanced MR imaging studies have demonstrated leakage of intravenous contrast agent from primary CNS neoplasms into peritumoral tissues and/or peritumor- al cysts. Because Gd-DTPA is a hydrophilic MR imaging contrast agent that remains exclusively within the CNS vasculature unless there is disruption of the blood-brain barrier, imaging evidence of agent extravasation is indicative of abnormal CNS vessel permeability. Bitzer and colleagues ${ }^{5}$ prospectively performed delayed $(0.6,3.5$, and 6.5 hours after Gd-DTPA injection) contrast-enhanced MR imaging studies in 28 patients with 32 meningiomas (12 without and 20 with associated peritumoral edema). Similar to findings of abnormal tumor vascular permeability underlying the formation of edema, they found significant contrast effusion (into the surrounding peritumoral tissue) from lesions with associated edema but not from those without edema. Relatedly, Lonser et al. ${ }^{28,32}$ analyzed contrast-enhanced MR imaging data obtained in 17 patients with 23 CNS hemangioblastomas that had associated edema and peritumoral cysts/syrinxes. They found that Gd-DTPA rapidly extravasates from highly permeable tumor vessels into not only surrounding edematous tissue, but also preferentially into peritumoral cysts (Fig. 1).

Most recently, long-term longitudinal MR imaging studies have directly demonstrated the pathological mechanisms and natural history of peritumoral cyst formation associated with CNS hemangioblastomas. Lonser and associates $^{32}$ performed serial MR imaging (T1-weighted with contrast and T2-weighted) in 22 patients with von Hippel-Lindau syndrome and demonstrated that hemangioblastoma-associated peritumoral edema progressed into peritumoral cysts (Figs. 2 and 3) over a period of several years (mean time from edema development to peritumoral cyst formation, 3 years; range 8-72 months). All peritumoral cysts were preceded by edema formation. These authors found that the peritumoral cysts grew significantly faster than the associated hemangioblastoma, indicating that tumor growth is not a necessary feature of peritumoral cyst formation. Moreover, the mass effect from these peritumoral cysts, not the underlying hemangioblastomas, was the cause of clinical symptoms in the patients.

Tumor Vessel Permeability. The intratumoral expression of VEGF ${ }^{4,8,44,51}$ by a variety of neoplasms has been positively correlated with the presence of edema and cysts and has been implicated as a factor that mediates the develop- 

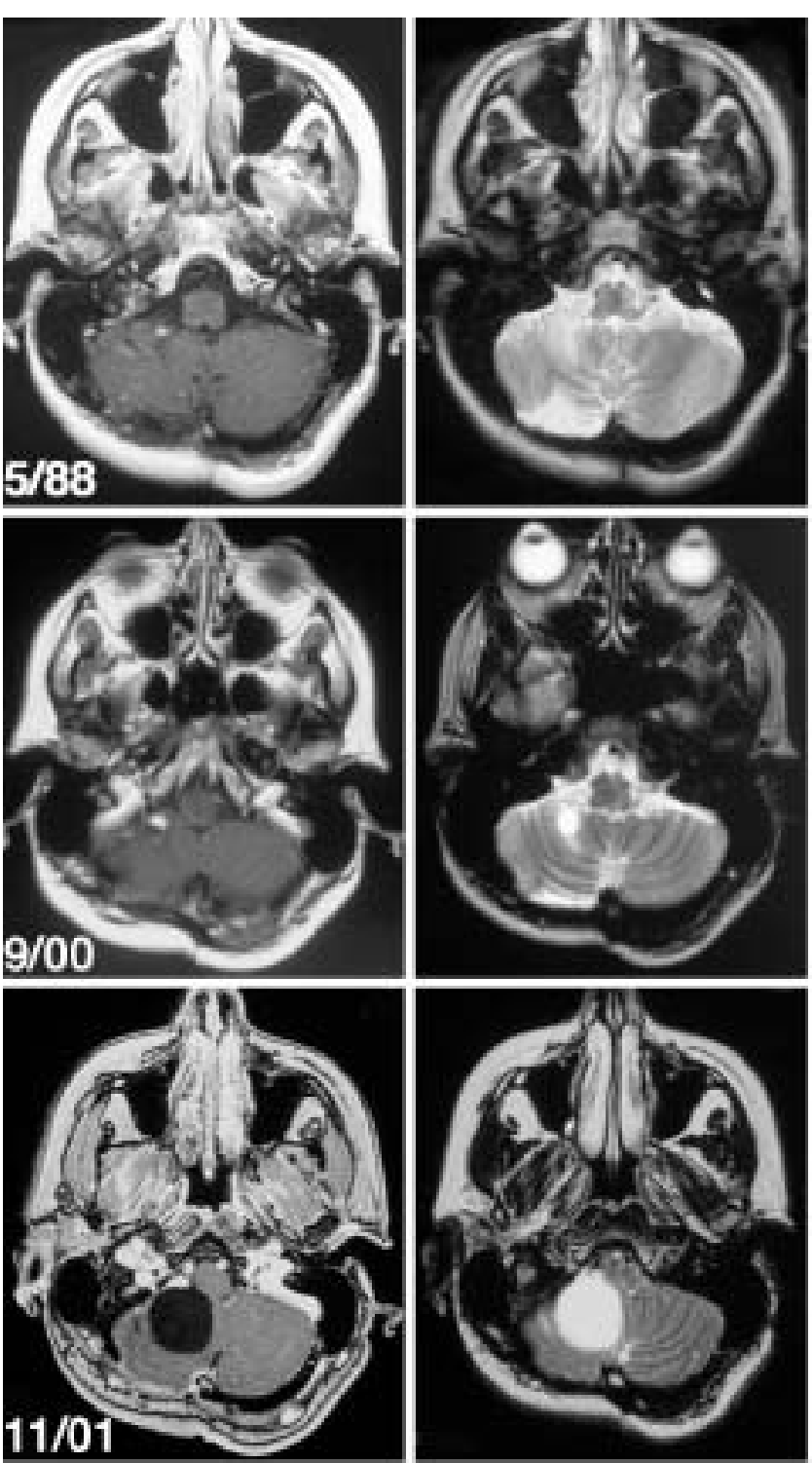

FIG. 2. Serial axial images obtained through the posterior fossa in a patient with a hemangioblastoma, demonstrating the progression of peritumoral edema to cyst formation. May 1998: Contrastenhanced T1-weighted MR image (left) revealing a hemangioblastoma in the right cerebellar hemisphere that was not initially associated with a cyst. A T2-weighted MR image (right) demonstrating peritumoral edema. September 2000: Contrast-enhanced T1-weighted MR image (left) exhibiting the right cerebellar hemangioblastoma with early cyst formation. A T2-weighted MR image (right) demonstrating peritumoral edema and a small cyst. November 2001: Contrast-enhanced T1-weighted MR image revealing the stable right cerebellar hemangioblastoma but a rapidly enlarging cyst. A T2-weighted MR image (right) showing the large associated cyst.

ment of peritumoral edema and cysts. ${ }^{12,52}$ Previously, it had been hypothesized that the association of VEGF expression with peritumoral edema and cysts was a result of VEGF's potent angiogenic and permeability-inducing actions on immediately surrounding tissue and vessels. However, a recent analysis ${ }^{47,50,57}$ of VEGF concentrations in the peritumoral cyst fluid of astrocytomas and heman- gioblastomas revealed that intracystic VEGF levels were far below (often more than an order of magnitude less) those necessary to cause increased permeability in cerebral $^{55}$ or cutaneous ${ }^{13}$ endothelial cells. In fact, some of these fluids had VEGF concentrations that were no higher than those in normal serum or plasma. These findings indicated that tumor vessels, which are in direct contact with the VEGF-producing stromal cells and are clearly abnormal/proliferative, are more susceptible to VEGF-induced leakiness than are vessels in the normal brain adjacent to the cyst, which are normal in appearance and exposed to only low concentrations of VEGF. Consistent with increased tumor vascular permeability driven by a VEGF-derived mechanism (through the overexpression of, or abnormal, VEGF receptors and/or increased tumor VEGF expression), Batchelor et al. ${ }^{3}$ found that the abnormal permeability of GBM tumor vessels normalized after systemic treatment with a pan-VEGF receptor inhibitor (AZD2171). These results lend further support to a process of peritumoral cyst formation and growth that is driven by the lesion itself.

Peritumoral Cyst Fluid Analysis. To confirm that peritumoral cyst fluid is derived from plasma extravasation through permeable tumor vessels, authors of a number of studies have characterized peritumoral cyst fluid proteins. ${ }^{11,24,25,32}$ These authors have demonstrated that peritumoral cyst fluid from gliomas, metastases, and other CNS tumors have a spectrum of proteins nearly identical to those found in serum plasma, indicating that a plasma ultrafiltrate is the source of edema and peritumoral cyst fluid. ${ }^{26,27}$ Lohle and associates ${ }^{24}$ investigated peritumoral cyst fluid from 22 patients with cerebral gliomas and found that $92 \%$ of the cyst fluid consisted of the plasma protein fraction. Later, Lohle et al. ${ }^{25}$ analyzed 39 cyst fluid samples from patients with primary and metastatic tumors and confirmed that $86 \%$ of the proteins in these cysts were plasma proteins. Most recently, Lonser et al..$^{32}$ and Glasker et al. ${ }^{11}$ performed 2D gel electrophoresis on hemangioblastoma peritumoral cyst fluid and found that the proteomic pattern of the cyst fluid and serum was similar among patients, indicating that the cyst fluid originated from a plasma ultrafiltrate through permeable vessels.

Hydrodynamic Forces. The rapid extravasation and interstitial spread (much greater and faster than would be expected if driven by diffusional forces alone) of $\mathrm{Gd}$ DPTA on MR imaging ${ }^{5,28,32}$ into regions of peritumoral edema and cysts suggests that vascular leakiness alone may not be sufficient for the significant development of edema and peritumoral cysts. Rather, it appears that pulsatile forces (hydrodynamic) combined with vascular leakiness may underlie widespread edema formation and peritumoral cyst formation. ${ }^{32}$ In 1984 Rennels et al. ${ }^{42}$ reported that horseradish peroxidase rapidly moves into the CNS parenchyma from the CSF and that this rapid movement occurs only if the normal pulsatile pressure waves in the CSF are present. In 1994, Oldfield et al. ${ }^{37}$ proposed that exaggerated pulse pressure in the spinal subarachnoid space associated with partial entrapment of the spinal CSF in patients with a Chiari malformation Type I was the driving force underlying the development of syringomyelia. In 1996 Stoodley et al. ${ }^{48}$ confirmed the observations of Rennels et al. ${ }^{42}$ and demonstrated that the bulk flow move- 
ment of macromolecules from the CSF into the spine by pulsatile CSF forces was directed to the central canal. Elevated mean and pulse pressures in the spinal CSF in patients with Chiari malformation Type I and syringomyelia was demonstrated by Heiss et al. ${ }^{14}$ in 1999. This series of observations supports a hypothesis that arterially-derived pulsations can enhance the movement of bulk flow into the CNS parenchyma and that under these conditions the excess extracellular fluid (seen as edema on MR images) can coalesce into a cystic cavity in the spinal cord and brain. $32,34,37,53,56$

\section{Clinical Implications}

\section{Predictors of Cyst Formation and Propagation}

The formation of peritumoral edema is indicative of possible peritumoral cyst formation. The accumulation of increased interstitial fluid (edema) occurs because the rate of fluid formation exceeds the rate of fluid removal. With progressive edema and arterial-driven pulsations, excess fluid generated by the lesion is distributed into surrounding tissue, creating an increase in interstitial pressure. , $17,32^{-12}$ As the surrounding tissue swells, it is subject to solid stresses (stretching) that favor cyst formation. Interstitial flow patterns probably change once a cyst forms, with the majority of excess interstitial fluid channeling into the adjacent cyst, given that this pathway provides the least flow resistance..$^{32}$ Initial accommodations to a change in this flow pattern can account for the initial rapid increases in cyst volume that have been observed (Figs. 2 and 3). ${ }^{32}$ Two physiological processes can also serve to stabilize cyst volume. First, as the cyst increases in size, its resorptive surface area increases its capacity to resorb excess fluid formation. Second, compression of adjacent parenchyma increases the interstitial pressure, directly opposing the hydrodynamic forces that drive fluid into the cyst.

Although the precise latency from edema to cyst formation cannot be determined, previous study data have indicated that it ranges from several months to several years. The long latency between edema formation and peritumoral cyst development may explain the relative lack of peritumoral cysts associated with very malignant CNS tumors (for example, GBM) compared with benign neoplasms (for example, hemangioblastomas). ${ }^{24,26,32} \mathrm{Be}-$ cause malignant tumors grow rapidly and are associated with mass effect over a short time course (daysweeks), it is quite possible that treatment or death due to these neoplasms occurs before peritumoral cysts form. Alternatively, benign slow-growing lesions that have significant peritumoral edema can develop peritumoral cysts before discovery and subsequently are associated with a higher incidence.

\section{Therapeutic Variables}

Implications for Familial Neoplasia Syndromes. Neurofibromatosis, Li-Fraumeni cancer syndrome, tuberous sclerosis, and von Hippel-Lindau syndrome are some familial neoplasia conditions that have well-described associated CNS tumors that can develop peritumoral cysts. ${ }^{15,29,35}$ Because associated peritumoral cysts can stop growing once the surface area of the sac is sufficiently large to resorb excess fluid extravasated from the tumor, they can become quiescent and potentially remain asymptomatic for long periods of time or even indefinitely. ${ }^{29,32,53}$ Thus, neuroimaging-demonstrated development or growth of peritumoral cysts is not an absolute indication for tumor resection in the context of familial neoplasia syndromes in which multiple known CNS lesions arise (benign tumors with peritumoral cysts), and judicious surgical treatment is critical because of the multiplicity of tumors and the potential for multiple surgeries during a patient's lifetime.

Implications for Surgery. Because increased tumor vascular permeability underlies peritumoral edema and subsequent cyst formation, resolution of peritumoral edema and cysts will occur after removal of the tumor that underlies their formation. Subsequently, removal of the cyst wall or wide fenestration of the cyst is not necessary, ${ }^{32,56}$ and peritumoral edema and cyst resolution (or cyst collapse) should occur after complete resection of the associated tumor. Thus, postoperative imaging evidence of peritumoral cyst propagation may be associated with residual tumor or another unresected tumor. ${ }^{34}$

Implications for Radiation. Animal and clinical study data have demonstrated that radiation at least transiently increases vascular permeability. ${ }^{9,21,40,41,58,59}$ Correspondingly, a number of authors have shown that after radiation of benign and malignant CNS tumors, there is an increase in peritumoral edema and de novo formation and/or propagation of peritumoral cysts. $718,36,45,46,49,54$ The observation that increased vascular permeability leads to the initiation and propagation of peritumoral edema (and, ultimately, to cysts) can explain why increased edema and peritumoral cyst formation have been associated with radiation. These findings also suggest that caution should be used when irradiating tumors with significant peritumoral edema or cysts because radiation-induced increases in tumor vessel permeability could lead to clinical deterioration.

Implications for Chemotherapy. Based on a process of peritumoral cyst formation that depends on increased tumor vascular permeability, chemotherapeutic agents that directly or indirectly decrease vascular permeability may have the benefit of reducing edema and/or peritumoral cyst formation, leading to improvement in edema- and/or cyst-related symptoms. Batchelor and colleagues ${ }^{3}$ administered AZD2171, a potent oral pan-VEGF receptor tyrosine kinase inhibitor, to 16 patients with recurrent GBM. These authors demonstrated with MR imaging that tumor vascular permeability normalization occurred within 24 hours of administering the drug and lasted at least 28 days, resulting in a significant reduction in peritumoral edema. Moreover, Aiello et al. ${ }^{2}$ and Girmens et al. ${ }^{10}$ have described patients with von Hippel-Lindau syndrome and loss of visual acuity due to macular hemangioblastomas that were treated with SU5416, another VEGF tyrosine kinase inhibitor. Although the treatment failed to decrease the tumor size, it did reduce cystoid macular edema and therefore improved visual acuity. Similar chemotherapeutic management paradigms may be feasible in patients with tumors not amenable to resection or radiation therapy but in whom clinical signs and symptoms are attributable to edema or peritumoral cysts. 

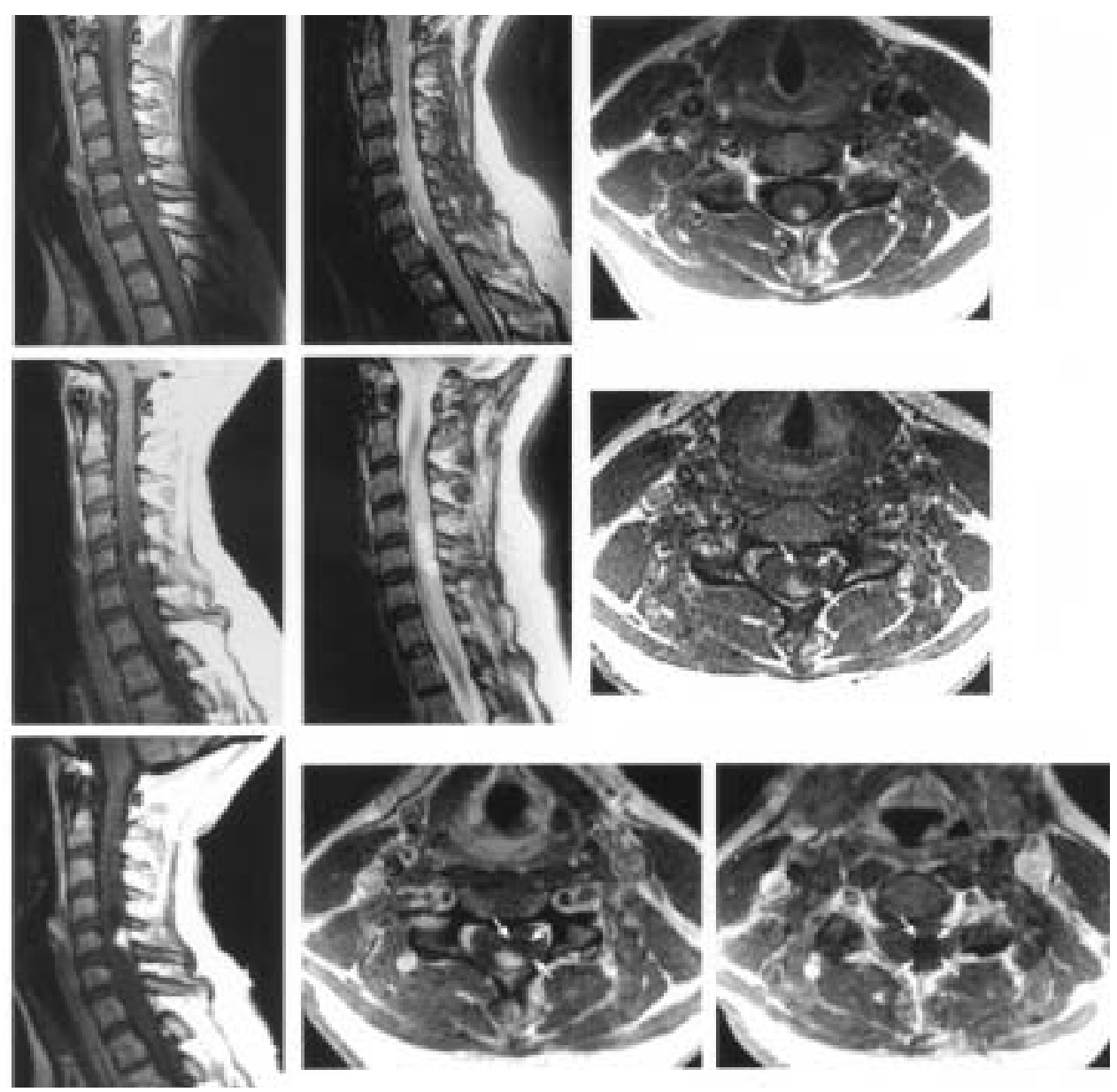

FIG. 3. Serial images obtained through the cervical spine in a patient during a 67-month period, demonstrating progression of edema associated with a spinal cord (C-5) hemangioblastoma into a syrinx. Upper row (March 1996): Midsagittal contrast-enhanced T1-weighted MR image (left) exhibiting a posterior C-5 hemangioblastoma with surrounding edema. Midsagittal T2-weighted MR image (center) depicting cervical edema surrounding the hemangioblastoma, but no cyst. Axial contrast-enhanced T1-weighted MR image (right) revealing the C-5 hemangioblastoma. Center row (December 1999): Midsagittal contrast-enhanced T1-weighted MR image (left) showing growth of the C-5 hemangioblastoma and early cyst formation. Midsagittal T2-weighted MR image (center) demonstrating the progression of cervical edema and development of an associated cyst. Axial contrast-enhanced T1-weighted MR image (right) displaying cyst formation (arrows) adjacent to the posterior C-5 hemangioblastoma. Lower row (October 2001): Midsagittal contrast-enhanced T1-weighted MR image showing enlargement of the C-5 hemangioblastoma-associated cyst. Axial contrast-enhanced T1-weighted MR image (center) through the C-5 hemangioblastoma showing the adjacent cyst (arrows) and maximum syrinx (right, arrows) diameter at the fourth cervical level.

Implications for Convection-Enhanced Delivery. Previously, we and others have shown that convection-enhanced delivery can be used to bypass the blood-brain barrier to distribute small- and large-molecular-weight compounds within the CNS safely and homogeneously over a wide range of volumes. ${ }^{6,19,20,31,38,43}$ Substrate is delivered under continuous pressure using bulk flow to drive solute through the interstitial space. Because the infusate is driven by a pressure gradient, convection-enhanced delivery increases the interstitial fluid content in the region of infusion and expands the interstitial space (edema) by the delivery of fluid at rates that can exceed the absorptive capacity of the tissue. Although we have not seen irreversible neurological deficits or significant elevations in intracranial pressure related to an increase in interstitial fluid with convective delivery in small animals, ${ }^{6,22}$ nonhu- man primates, ${ }^{30,33}$ or humans, ${ }^{20,31}$ it is conceivable that sustained interstitial delivery of infusate can create a situation in which delivery overwhelms the tissue's absorptive capacity, leading to edema formation and to subsequent cyst development. Thus, an interstitial infusion delivered over an extended interval, as might be necessary for the successful delivery of neurotrophic factors, could lead to cyst formation. In that circumstance, the cessation of, a rate reduction in, or the use of intermittent intervals of infusion should prevent or lead to resolution of edema, cysts, and symptoms.

\section{Conclusions}

Edema is a common precursor to peritumoral cyst formation in the CNS. The mechanism of cyst formation in 
patients with CNS neoplasms appears to be the result of an interstitial process that starts with the generation of edema. Vascular permeability factors acting locally on abnormal tumor vasculature in conjunction with hydrodynamic forces drive fluid (plasma) extravasation. When these forces overcome the ability of the surrounding tissue to resorb fluid, edema (with its associated increased interstitial pressure) and subsequent cyst formation occur. Because the tumor itself, not the adjacent tissue, is the source of the edema that precedes cyst formation, tumor resection is sufficient to result in the resolution of edema and cysts by eliminating the underlying pathophysiological process. Similarly, chemotherapeutic management paradigms that reduce tumor vascular permeability may be helpful in patients with tumors not amenable to resection or radiation therapy, but in whom clinical signs and symptoms are attributable to edema or peritumoral cysts.

\section{References}

1. Afra D, Norman D, Levin VA: Cysts in malignant gliomas. Identification by computerized tomography. J Neurosurg 53: $821-825,1980$

2. Aiello LP, George DJ, Cahill MT, Wong JS, Cavallerano J, Hannah AL, et al: Rapid and durable recovery of visual function in a patient with von hippel-lindau syndrome after systemic therapy with vascular endothelial growth factor receptor inhibitor su5416. Ophthalmology 109:1745-1751, 2002

3. Batchelor TT, Sorensen AG, di Tomaso E, Zhang WT, Duda DG, Cohen KS, et al: AZD2171, a pan-VEGF receptor tyrosine kinase inhibitor, normalizes tumor vasculature and alleviates edema in glioblastoma patients. Cancer Cell 11:83-95, 2007

4. Berkman RA, Merrill MJ, Reinhold WC, Monacci WT, Saxena A, Clark WC, et al: Expression of the vascular permeability factor/vascular endothelial growth factor gene in central nervous system neoplasms. J Clin Invest 91:153-159, 1993

5. Bitzer M, Nagele T, Geist-Barth B, Nagele T, Geist-Barth B, Klose U, et al: Role of hydrodynamic processes in the pathogenesis of peritumoral brain edema in meningiomas. J Neurosurg 93:594-604, 2000

6. Bobo RH, Laske DW, Akbasak A, Morrison PF, Dedrick RL, Oldfield EH: Convection-enhanced delivery of macromolecules in the brain. Proc Natl Acad Sci U S A 91:2076-2080, 1994

7. Boethius J, Ulfarsson E, Rahn T, Lippittz B: Gamma knife radiosurgery for pilocytic astrocytomas. J Neurosurg 97 (5 Suppl):677-680, 2002

8. Bruce JN, Criscuolo GR, Merrill MJ, Moquin RR, Blacklock JB, Oldfield EH: Vascular permeability induced by protein product of malignant brain tumors: inhibition by dexamethasone. J Neurosurg 67:880-884, 1987

9. Cao Y, Tsien CI, Shen Z, Tatro DS, Ten Haken R, Kessler ML, et al: Use of magnetic resonance imaging to assess bloodbrain/blood-glioma barrier opening during conformal radiotherapy. J Clin Oncol 23:4127-4136, 2005

10. Girmens JF, Erginay A, Massin P, Scigalla P, Gaudric A, Richard S: Treatment of von Hippel-Lindau retinal hemangioblastoma by the vascular endothelial growth factor receptor inhibitor SU5416 is more effective for associated macular edema than for hemangioblastomas. Am J Ophthalmol 136: 194-196, 2003

11. Glasker S, Vortmeyer AO, Lonser RR, Lubensky IA, Okamoto $\mathrm{H}$, Xia JB, et al: Proteomic analysis of hemangioblastoma cyst fluid. Cancer Biol Ther 5:549-553, 2006

12. Goldman CK, Bharara S, Palmer CA, Vitek J, Tsai JC, Weiss $\mathrm{HL}$, et al: Brain edema in meningiomas is associated with increased vascular endothelial growth factor expression. Neurosurgery 40:1269-1277, 1997

13. Heiss JD, Papavassiliou E, Merrill MJ, Nieman L, Knightly JJ, Walbridge S, et al: Mechanism of dexamethasone suppression of brain tumor-associated vascular permeability in rats. Involvement of the glucocorticoid receptor and vascular permeability factor. J Clin Invest 98:1400-1408, 1996

14. Heiss JD, Patronas N, DeVroom HL, Shawker T, Ennis R, Kammerer W, et al: Elucidating the pathophysiology of syringomyelia. J Neurosurg 91:553-562, 1999

15. Hemminki K, Li X: Familial risks in nervous system tumors. Cancer Epidemiol Biomarkers Prev 12:1137-1142, 2003

16. Holodny AI, Nusbaum AO, Festa S, Pronin IN, Lee HJ, Kalnin $\mathrm{AJ}$ : Correlation between the degree of contrast enhancement and the volume of peritumoral edema in meningiomas and malignant gliomas. Neuroradiology 41:820-825, 1999

17. Jain RK, Baxter LT: Mechanisms of heterogeneous distribution of monoclonal antibodies and other macromolecules in tumors: significance of elevated interstitial pressure. Cancer Res 48: 7022-7032, 1988

18. Kida Y, Kobayashi T, Mori Y: Gamma knife radiosurgery for low-grade astrocytomas: results of long-term follow up. J Neurosurg 93 (3 Suppl):42-46, 2000

19. Kunwar S, Chang SM, Prados MD, Berger MS, Sampson JH, Croteau D, et al: Safety of intraparenchymal convection-enhanced delivery of cintredekin besudotox in early-phase studies. Neurosurg Focus 20(4):E15, 2006

20. Laske DW, Youle RJ, Oldfield EH: Tumor regression with regional distribution of the targeted toxin TF-CRM107 in patients with malignant brain tumors. Nat Med 3:1362-1368, 1997

21. Lee MC, Cha S, Chang SM, Nelson SJ: Dynamic susceptibility contrast perfusion imaging of radiation effects in normalappearing brain tissue: changes in the first-pass and recirculation phases. J Magn Reson Imaging 21:683-693, 2005

22. Lieberman DM, Laske DW, Morrison PF, Bankiewicz KS, Oldfield EH: Convection-enhanced distribution of large molecules in gray matter during interstitial drug infusion. J Neurosurg 82:1021-1029, 1995

23. Lohle PN, van Mameren H, Zwinderman KH, Teepen HL, Go KG, Wilmink JT: On the pathogenesis of brain tumor cysts: a volumetric study of tumor, oedema and cyst. Neuroradiology 42:639-642, 2000

24. Lohle PN, Verhagen IT, Teelken AW, Blaauw EH, Go KG: The pathogenesis of cerebral gliomatous cysts. Neurosurgery 30: 180-185, 1992

25. Lohle PN, Wurzer HA, Seelen PJ, Kingma LM, Go KG: Analysis of fluid in cysts accompanying various primary and metastatic brain tumors: proteins, lactate and $\mathrm{pH}$. Acta Neurochir (Wien) 140:14-19, 1998

26. Lohle PN, Wurzer HA, Seelen PJ, Kingma LM, Go KG: Cystic lesions accompanying extra-axial tumors. Neuroradiology 41:13-17, 1999

27. Lohle PN, Wurzer HA, Seelen PJ, Kingma LM, Go KG: The pathogenesis of cysts accompanying intra-axial primary and metastatic tumors of the central nervous system. J Neurooncol 40:277-285, 1998

28. Lonser RR, Butman JA, Oldfield EH: Pathogenesis of tumorassociated syringomyelia demonstrated by peritumoral contrast material leakage. Case illustration. J Neurosurg Spine 4:426, 2006

29. Lonser RR, Glenn GM, Walther M, Chew EY, Libutti SK, Linehan WM, et al: von Hippel-Lindau disease. Lancet 361: 2059-2067, 2003

30. Lonser RR, Gogate N, Morrison PF, Wood JD, Oldfield EH: Direct convective delivery of macromolecules to the spinal cord. J Neurosurg 89:616-622, 1998

31. Lonser RR, Schiffman R, Robison RA, Butman JA, Quezado Z, Walker ML, et al: Image-guided, direct convective delivery of 
glucocerebrosidase for neuronopathic Gaucher disease. Neurology 68:254-261, 2007

32. Lonser RR, Vortmeyer AO, Butman JA, Glasker S, Finn MA, Ammerman JM, et al: Edema is a precursor to central nervous system peritumoral cyst formation. Ann Neurol 58:392-399, 2005

33. Lonser RR, Walbridge S, Garmestani K, Butman JA, Walters HA, Vortmeyer AO, et al: Successful and safe perfusion of the primate brainstem: in vivo magnetic resonance imaging of macromolecular distribution during infusion. J Neurosurg 97: 905-913, 2002

34. Lonser RR, Weil RJ, Wanebo JE, DeVroom HL, Oldfield EH: Surgical management of spinal cord hemangioblastomas in patients with von Hippel-Lindau disease. J Neurosurg 98: 106-116, 2003

35. Melean G, Sestini R, Ammannati F, Papi L: Genetic insights into familial tumors of the nervous system. Am J Med Genet C Semin Med Genet 129:74-84, 2004

36. Niemela M, Lim YJ, Soderman M, Jaaskelainen J, Lindquist C: Gamma knife radiosurgery in 11 hemangioblastomas. J Neurosurg 85:591-596, 1996

37. Oldfield EH, Muraszko K, Shawker TH, Patronas NJ: Pathophysiology of syringomyelia associated with Chiari I malformation of the cerebellar tonsils. Implications for diagnosis and treatment. J Neurosurg 80:3-15, 1994

38. Patel SJ, Shapiro WR, Laske DW, Jensen RL, Asher AL, Wessels BW, et al: Safety and feasibility of convection-enhanced delivery of Cotara for the treatment of malignant glioma: initial experience in 51 patients. Neurosurgery 56:1243-1253, 2005

39. Pronin IN, Holodny AI, Petraikin AV: MRI of high-grade glial tumors: correlation between the degree of contrast enhancement and the volume of surrounding edema. Neuroradiology 39:348-350, 1997

40. Qin D, Ma J, Xiao J, Tang Z: Effect of brain irradiation on blood-CSF barrier permeability of chemotherapeutic agents. Am J Clin Oncol 20:263-265, 1997

41. Qin D, Ou G, Mo H, Song Y, Kang G, Hu Y, et al: Improved efficacy of chemotherapy for glioblastoma by radiation-induced opening of blood-brain barrier: clinical results. Int J Radiat Oncol Biol Phys 51:959-962, 2001

42. Rennels ML, Gregory TF, Blaumanis OR, Fujimoto K, Grady PA: Evidence for a 'paravascular' fluid circulation in the mammalian central nervous system, provided by the rapid distribution of tracer protein throughout the brain from the subarachnoid space. Brain Res 326:47-63, 1985

43. Sampson JH, Akabani G, Archer GE, Bigner DD, Berger MS, Friedman AH, et al: Progress report of a Phase I study of the intracerebral microinfusion of a recombinant chimeric protein composed of transforming growth factor (TGF)-alpha and a mutated form of the Pseudomonas exotoxin termed PE-38 (TP38 ) for the treatment of malignant brain tumors. J Neurooncol 65:27-35, 2003

44. Senger DR, Galli SJ, Dvorak AM, Perruzzi CA, Harvey VS, Dvorak HF: Tumor cells secrete a vascular permeability factor that promotes accumulation of ascites fluid. Science 219: 983-985, 1983

45. Shuto T, Inomori S, Fujino H, Nagano H, Hasegawa N, Kakuta Y: Cyst formation following gamma knife surgery for intracranial meningioma. J Neurosurg 102 Suppl:134-139, 2005

46. Simonova G, Novotny J Jr, Liscak R: Low-grade gliomas treated by fractionated gamma knife surgery. J Neurosurg 102 Suppl:19-24, 2005
47. Stockhammer G, Obwegeser A, Kostron H, Schumacher P, Muigg A, Felber S, et al: Vascular endothelial growth factor (VEGF) is elevated in brain tumor cysts and correlates with tumor progression. Acta Neuropathol 100:101-105, 2000

48. Stoodley MA, Jones NR, Brown CJ: Evidence for rapid fluid flow from the subarachnoid space into the spinal cord central canal in the rat. Brain Res 707:155-164, 1996

49. Tago M, Terahara A, Shin M, Maruyama K, Kurita H, Nakagawa K, et al: Gamma knife surgery for hemangioblastomas. J Neurosurg 102 Suppl:171-174, 2005

50. Takano S, Yoshii Y, Kondo S, Suzuki H, Maruno T, Shirai S, et al: Concentration of vascular endothelial growth factor in the serum and tumor tissue of brain tumor patients. Cancer Res 56:2185-2190, 1996

51. Toi M, Matsumoto T, Bando H: Vascular endothelial growth factor: its prognostic, predictive, and therapeutic implications. Lancet Oncol 2:667-673, 2001

52. Vaquero J, Zurita M, Oya S, Coca S, Salas C: Vascular permeability factor expression in cerebellar hemangioblastomas: correlation with tumor-associated cysts. J Neurooncol 41:3-7, 1999

53. Wanebo JE, Lonser RR, Glenn GM, Oldfield EH: The natural history of hemangioblastomas of the central nervous system in patients with von Hippel-Lindau disease. J Neurosurg 98: 82-94, 2003

54. Wang EM, Pan L, Wang BJ, Zhang N, Zhou LF, Dong YF, et al: The long-term results of gamma knife radiosurgery for hemangioblastomas of the brain. J Neurosurg 102 Suppl: 225-229, 2005

55. Wang W, Merrill MJ, Borchardt RT: Vascular endothelial growth factor affects permeability of brain microvessel endothelial cells in vitro. Am J Physiol 271:C1973-C1980, 1996

56. Weil RJ, Lonser RR, DeVroom HL, Wanebo JE, Oldfield EH: Surgical management of brainstem hemangioblastomas in patients with von Hippel-Lindau disease. J Neurosurg 98: 95-105, 2003

57. Weindel K, Moringlane JR, Marmé D, Weich HA: Detection and quantification of vascular endothelial growth factor/vascular permeability factor in brain tumor tissue and cyst fluid: the key to angiogenesis? Experimental study. Neurosurgery 35: 439-449, 1994

58. Yuan H, Gaber MW, Boyd K, Wilson CM, Kiani MF, Merchant TE: Effects of fractionated radiation on the brain vasculature in a murine model: blood-brain barrier permeability, astrocyte proliferation, and ultrastructural changes. Int J Radiat Oncol Biol Phys 66:860-866, 2006

59. Yuan H, Gaber MW, McColgan T, Naimark MD, Kiani MF, Merchant TE: Radiation-induced permeability and leukocyte adhesion in the rat blood-brain barrier: modulation with antiICAM-1 antibodies. Brain Res 969:59-69, 2003

Manuscript submitted February 15, 2007.

Accepted March 23, 2007.

This research was supported by the Intramural Research Program, National Institute of Neurological Disorders and Stroke, National Institutes of Health.

Address reprint requests to: Russell R. Lonser, M.D., Surgical Neurology Branch, National Institute of Neurological Disorders and Stroke, National Institutes of Health, 10 Center Drive, Building 10, Room 5D37, Bethesda, Maryland 20892-1414. email: lonserr@ ninds.nih.gov. 